linked by a common source of transmission or a shared behaviour and that this link could be demonstrated by phylogenetic analysis.

Methods A case definition of men, aged 35-75, presenting with acute HBV, with no recorded risk factors, was adopted. The PHE Database 'HP zone' was used to compile a line list of new acute HBV matching the case definition within the undisclosed county, from January 2012 to January 2015. Inclusion criteria for all cases required HBV core IgM positive status with clinical symptoms or biochemical markers consistent with acute hepatitis. Data on risk factors was compiled, as part of routine investigation, from the PHE acute HBV questionnaire using open and focussed questioning. Serum specimens on the line list were sent to the PHE laboratory, Colindale for phylogenetic analysis.

Results 33 cases of acute HBV were identified with 19 men fitting the case definition. The line list was expanded to include the remaining 7 men aged $35-75$ in the same region and timeframe who did report risk factors for HBV exposure (total=26). 6 cases did not have stored serum for genotyping. 17 cases of HBV Genotype A2 were identified, 14 met the exact case definition with no risk factors with the remaining 3 reporting high-risk sexual activity with men. 3 Cases of Genotype D were identified, all of which reported risk factors. 14 of the 19 cases that fitted the definition described themselves as heterosexual and married at the time of diagnosis. All spouses tested negative for HBV.

Of the 17 A2 genotypes 16 were 99\%-100\% identical and 12 were $99.7 \%-100 \%$ identical at the nucleotide level with one case differing by a single base pair at the HBsAg region. All cases were of a single, stable strain known as the 'Prisoner Variant', which has increased in prevalence from 1990, primarily in the prison population of north England.

Conclusions The genotypic link of at least 16 cases with a non-prevalent strain of $\mathrm{HBV}$ indicates a common source of infection. The lack of confirmed risk factors in so many cases may indicate a shift in the 'epi-picture' with new behaviours making at-risk groups harder to identify for targeted healtheducation or vaccination. The cluster remains active with further cases being investigated.

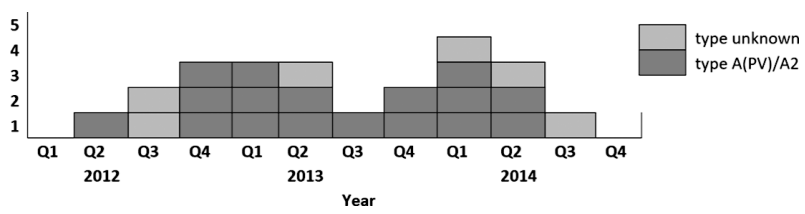

Abstract PTH-112 Figure 1 Epidemic curve displaying cases of acute Hep B per quarter 2012 to 2015; cases other than A/A2 excluded

\section{PTH-113 THE SUCCESS OF KAFFES STENT INSERTIONS FOR POST LIVER TRANSPLANT ANASTOMOTIC STRICTURES}

Deepak Joshi* ${ }^{*}$ Ben Warner*, Phillip Harrison, John Devlin, David Reffitt, Yasser El-Sherif, Shirin Khorsandi, Andreas Prachalias, Miriam Cortes Cerisuelo, Krishna Menon, Wayel Jassem, Parthi Srinivasan, Hector Vilca-Melendez, Michael Heneghan, Nigel Heaton. Institute of Liver Studies, King's College Hospital, London, UK

\subsection{6/gutjnl-2018-BSGAbstracts.269}

Introduction Biliary anastomotic strictures (AS) occur in around $30 \%$ of patients following liver transplantation and are treated by endoscopic dilatation and plastic stent (PS) insertion. However, AS frequently recur and patients require multiple procedures. The Kaffes stent (KS, Taewoong Medical) is a removable, covered metal stent designed to be deployed across AS.

Methods To examine outcomes in patients with AS, we compared a recent cohort of patients treated using KS with a historical cohort of patients who received PS.

Results The 22 patients (12 females) treated by KS had mean age 55 (range 22-69) years; 11 patients had DBD and 11 DCD grafts; mean cold ischaemia time was 9.6 \pm 3.3 hours. Four patients had failed previous treatment with PS. To date, 16 patients have had KS removed. The 69 patients $(20$ female) treated by PS were similar, mean age 51 (range 2879) years; 47 patients had DBD and 22 DCD grafts; mean cold ischaemia time was $8.9 \pm 3.1$ hours.

AS resolved after one deployment of KS in 14 out of 16 patients (88\%) compared to 26 out of 69 patients (38\%) receiving their first PS (Relative Risk of persistent stricture (KS vs PS $)=0.2$, 95\% CI $0.05-0.74 ; \mathrm{p}=0.016$; number to treat by KS for one benefit $=2$, 95\% CI 1.3-4.0). There were no complications, including stent migration, after KS compared to $6(8.4 \%)$ in the PS group (3 cholangitis, 2 pancreatitis, and 1 bleeding). All KS were removed successfully, although 1 stent needed 2 attempts because of wire migration. Following initial ERCP, PS patients required more ERCPs (mean 2.71 vs 1.13 more; $\mathrm{p}<0.01$ ) and $32 \%$ required biliary reconstruction.

Conclusion Our data indicate that the $\mathrm{KS}$ is a promising Method for managing post-transplant AS because the majority of strictures are treated by deployment of a single stent at first ERCP.

\section{PTH-114 REAL-WORLD PERFORMANCE OF NON-INVASIVE IMAGING MODALITIES IN THE DETECTION OF COMMON BILE DUCT STONES (CBDS)}

Javed Iqbal* ${ }^{*}$ Benjamin Giles, Chris Palmer-Jones, William Crinnion, Mohammed Syed, Simon Phillpotts, Sudeep Tanwar. Whipps Cross University Hospital, Barts Health NHS Trust, London, UK

\subsection{6/gutjnl-2018-BSGAbstracts. 270}

Introduction Cholangiography at endoscopic retrograde cholangio-pancreatography (ERCP), the reference standard for detecting CBDS, is not practicable as the primary Method for identifying CBDS as it is invasive and has an associated morbidity. With regard to the non-invasive identification of CBDS, the current BGS guidance outlines pooled sensitivities of $73 \%$, $69 \%-87 \%$ and $93 \%$ for ultrasound (US), computed tomography (CT) and magnetic resonance cholangio-pancreatography (MRCP) respectively. In this study we report the real-world sensitivity of these imaging modalities to detect CBDS.

Method All cases of CBDS confirmed at ERCP over 12 months were identified prospectively. The imaging modalities employed prior to ERCP were identified and evaluated for the presence or absence of duct dilatation $(>6 \mathrm{~mm})$ or intraductal contents (stone or sludge) thereby defining the sensitivity of these parameters for detecting CBDS. 95\% confidence interval (CI) of the sensitivities was calculated.

Results In total, 102 patients had ERCP for CBDS (female $57 \%)$ after presenting with pain $(83 \%)$ and jaundice $(45 \%$. Liver function tests were abnormal in $82 \%$. 48 patients had CBDS $\geq 1 \mathrm{~cm}$ (maximum size $35 \mathrm{~mm}$, mean $9.6 \mathrm{~mm}$ ). All US scans in this study were performed by sonographers. 